\title{
STABILITY OF COUPLED SYSTEMS
}

\author{
FARID AMMAR KHODJA, ASSIA BENABDALLAH AND DJAMEL TENIOU
}

\begin{abstract}
The exponential and asymptotic stability are studied for certain coupled systems involving unbounded linear operators and linear infinitesimal semigroup generators. Examples demonstrating the theory are also given from the field of partial differential equations.
\end{abstract}

\section{INTRODUCTION}

In [1] and [2], the authors have studied a system which consists in a coupling of the wave equation with the heat equation. They showed that, under some conditions, one has exponential stability or asymptotic stability. Another system has been studied in [3]. Stability results are also proved there. Our goal, in this paper, is to give results for the stability of more general systems than those cited.

The system we want to study is:

$$
\left\{\begin{array}{l}
u^{\prime}=A u+B v \\
v^{\prime}=-B^{*} u+C v \\
u(0)=u_{0}, \quad v(0)=v_{0} .
\end{array}\right.
$$

where $A, B$ and $C$ are unbounded operators on complex Hilbert spaces which will be precisely defined in what follows.

This work is divided into three parts. In the second section, sufficient conditions are given which insure the exponential stability of the semigroup associated to system (1). The third section is devoted to the study of the asymptotic stability. Applications to some examples end this paper.

\section{Exponential STABILITY}

In this section, we set the following assumption:

1991 Mathematics Subject Classification. Primary 35B35.

Key words and phrases. Complex Hilbert space, unbounded linear operators, coupled systems, infinitesimal semigroup generators, exponential and asymptotic stability.

This work was done while the third author was visiting the Laboratoire de Calcul Scientifique of the Besançon University.

Received: April 30, 1996. 


\section{Assumption (E.S)}

1) $(A, D(A))$ is a generator of an exponentially stable semigroup of contractions $\left(S_{A}(t)\right)$ on the Hilbert space $X$ : there exist $\omega_{A}>0$ and $M_{A}>0$ such that

$$
\left\|S_{A}(t)\right\|_{X} \leq M_{A} e^{-t \omega_{A}} \quad \forall t \geq 0 ;
$$

2) $(C, D(C))$ is self-adjoint on the Hilbert space $Y$ and generator of an exponentially stable semigroup $\left(S_{C}(t)\right)$;

3) $(B, D(B))$ is an operator from $Y$ to $X$ such that $D(A) \subset D\left(B^{*}\right)$ and it is $(-C)^{\frac{1}{2}}$ - bounded, i.e: $D\left((-C)^{\frac{1}{2}}\right) \subset D(B)$ and

$$
B(-C)^{-\frac{1}{2}} \in L(Y, X)
$$

4) The operator

$$
L=\left(\begin{array}{cc}
A & B \\
-B^{*} & C
\end{array}\right)
$$

of domain $D(A) \times D(C)$ is closed and there exists $\lambda_{0}>0$ such that $\left(\lambda_{0} I-L\right)$ is onto.

Proposition 1. Under the assumption (E.S), the operator $L$ is an infinitesimal generator of a semigroup $S_{L}(t)$ of contractions.

Proof. L is monotone since for all $(u, v) \in D(L)$, one has:

$$
\left\langle L\left[\begin{array}{l}
u \\
v
\end{array}\right],\left[\begin{array}{l}
u \\
v
\end{array}\right]\right\rangle_{X \times Y}=\langle A u, u\rangle_{X}+\langle B v, u\rangle_{X}-\left\langle B^{*} u, v\right\rangle_{Y}+\langle C v, v\rangle_{Y} .
$$

So, using assumptions (E.S 1$)-($ E.S 2$)$ :

$$
\operatorname{Re}\left\langle L\left[\begin{array}{l}
u \\
v
\end{array}\right],\left[\begin{array}{l}
u \\
v
\end{array}\right]\right\rangle_{X, Y}=\operatorname{Re}\left(\langle A u, u\rangle_{X}+\langle C v, v\rangle_{Y}\right) \leq 0 .
$$

The assumption $\left(E . S_{4}\right)$ implies that $L$ is maximal. The Lumer-Phillips theorem gives the result.

Then, for all $\left(u_{0}, v_{0}\right) \in D(L)$, the system

$$
\left\{\begin{array}{l}
Y^{\prime}(t)=L Y(t) \\
Y(0)=Y_{0}=\left[\begin{array}{l}
u_{0} \\
v_{0}
\end{array}\right]
\end{array}\right.
$$

has a unique solution $Y$ in $C\left(\left[0,+\infty[; D(L)) \cap C^{1}([0,+\infty[, X \times Y)[10]\right.\right.$.

Let us denote by $Y(t)=\left[\begin{array}{l}u(t) \\ v(t)\end{array}\right], t \geq 0$, a solution of (2). One has:

Theorem 2. The semigroup $S_{L}(t)$ generated by $(L, D(L))$ is exponentially stable: there exist $\omega>0$ and $M>0$ such that

$$
\left\|S_{L}(t)\right\|_{X \times Y} \leq M e^{-\omega t} \quad \forall t \geq 0 .
$$


Before proving the theorem, let us recall (see Pritchard and Zabczyk [11]) that a semigroup $(S(t))_{t}$ generated on a Hilbert space $\left(H,\langle,\rangle_{H}\right)$ by an operator $(A, D(A))$ is exponentially stable if and only if there exists a bounded, positive and selfadjoint operator $P$ on $H$ such that:

$$
2\langle P A Y, Y\rangle_{H}=-\|Y\|_{H}^{2} \quad \forall Y \in D(A) .
$$

This last equation (in $P$ ) is called the Lyapunov equation $(E . L)$ and the solution $P$ the Lyapunov operator. As the semigroups associated to $(A, D(A))$ and $(C, D(C))$ are exponentially stable, we denote by $P_{A}$ and $P_{C}$ their associated Lyapunov operators. One has:

$$
\begin{cases}2 \operatorname{Re}\left\langle P_{A} A u, u\right\rangle_{X}=-\|u\|_{X}^{2} & \forall u \in D(A), \\ 2 \operatorname{Re}\left\langle P_{C} C v, v\right\rangle_{Y}=-\|v\|_{Y}^{2} & \forall v \in D(C) .\end{cases}
$$

In fact, as the operator $(C, D(C))$ is selfadjoint and its semigroup exponentially stable, the associated Lyapunov operator $P_{C}$ is $-\frac{1}{2} C^{-1}$. So we have:

Lemma 3. There exists $\varepsilon_{0}>0$ such that for all $\varepsilon \leq \varepsilon_{0}$ and all $Y_{0} \in D(L)$, the functional

$$
\rho_{\varepsilon}(t)=\|Y(t)\|_{X \times Y}^{2}+\varepsilon\left\langle P_{A} u(t), u(t)\right\rangle_{X}+\varepsilon\left\langle-C^{-1} v(t), v(t)\right\rangle_{Y}
$$

is such that there exists $D(\varepsilon)>0$ for which

$$
\rho_{\varepsilon}(t) \leq \rho_{\varepsilon}(0) e^{-D(\varepsilon) t} \quad \text { for all } t \geq 0 .
$$

Proof. We will prove that, for sufficiently small $\varepsilon>0$, there exists a positive constant $D(\varepsilon)$ such that

$$
\frac{d}{d t} \rho_{\varepsilon}(t) \leq-D(\varepsilon) \rho_{\varepsilon}(t)
$$

Evaluating the derivative of $\rho_{\varepsilon}(t)$, we get from system (2)

$$
\frac{d}{d t}\|Y(t)\|_{X \times Y}^{2}=2 \operatorname{Re}\langle C v(t), v(t)\rangle_{Y}+2 \operatorname{Re}\langle A u(t), u(t)\rangle_{X}
$$

and from (3)

$$
\begin{aligned}
\frac{d}{d t}\left\langle P_{A} u(t), u(t)\right\rangle_{X} & =-\|u(t)\|_{X}^{2}+2 \operatorname{Re}\left\langle B v(t), P_{A} u(t)\right\rangle_{X}, \\
\frac{d}{d t}\left\langle C^{-1} v(t), v(t)\right\rangle_{Y} & =-\|v(t)\|_{Y}^{2}-\operatorname{Re}\left\langle B^{*} u(t), C^{-1} v(t)\right\rangle_{Y} .
\end{aligned}
$$

Now we estimate the products using the assumptions on $B$ and $C$ :

$$
\begin{aligned}
\left|\left\langle B v(t), P_{A} u(t)\right\rangle_{X}\right|= & \left|\left\langle B(-C)^{-\frac{1}{2}}(-C)^{\frac{1}{2}} v(t), P_{A} u(t)\right\rangle_{X}\right| \\
\leq & \frac{1}{2 \alpha}\left\|B(-C)^{-\frac{1}{2}}\right\|_{L(Y, X)}^{2}\left\|(-C)^{\frac{1}{2}} v(t)\right\|_{Y}^{2} \\
& +\frac{\alpha}{2}\left\|P_{A}\right\|_{L(X)}^{2}\|u(t)\|_{X}^{2},
\end{aligned}
$$

where $\alpha$ is a positive constant. We recall that the assumption (E.S $\left.\mathrm{S}_{3}\right)$ implies that all the quantities of the right member are bounded. 
Next, we estimate the last term:

$$
\begin{aligned}
\left|\left\langle B^{*} u(t), C^{-1} v(t)\right\rangle_{Y}\right|= & \left|\left\langle u(t), B(-C)^{-\frac{1}{2}}(-C)^{-1}(-C)^{\frac{1}{2}} v(t)\right\rangle_{Y}\right| \\
\leq & \frac{\alpha}{2}\|u(t)\|_{X}^{2}+\frac{1}{2 \alpha}\left\|B(-C)^{-\frac{1}{2}}\right\|_{L(Y, X)}^{2} \\
& \cdot\left\|(-C)^{-1}\right\|_{L(Y)}^{2}\left\|(-C)^{\frac{1}{2}} v(t)\right\|_{Y}^{2} .
\end{aligned}
$$

Grouping these estimates together (with the use of the dissipativity of $A$ ), we get

$$
\begin{aligned}
& \frac{d}{d t} \rho_{\varepsilon}(t) \leq-\varepsilon\|v(t)\|_{Y}^{2}-\varepsilon\left(1-\frac{\alpha}{2}\left\|P_{A}\right\|_{L(X)}^{2}-\frac{\alpha}{2}\right)\|u(t)\|_{X}^{2} \\
& \quad+\left[\frac{\varepsilon}{2 \alpha}\left\|B(-C)^{-\frac{1}{2}}\right\|_{L(Y, X)}^{2}\left(\left\|(-C)^{-1}\right\|_{L(Y)}^{2}+1\right)-2\right]\left\|(-C)^{\frac{1}{2}} v(t)\right\|_{Y}^{2} .
\end{aligned}
$$

If we choose $\alpha<\alpha_{0}$, where

$$
\alpha_{0}=2\left(\left\|P_{A}\right\|_{L(X)}^{2}+1\right)^{-1},
$$

and $\varepsilon<\varepsilon_{0}$, where

$$
\varepsilon_{0}=\frac{4 \alpha}{\left\|B(-C)^{-\frac{1}{2}}\right\|_{L(Y, X)}^{2}\left(\left\|(-C)^{-1}\right\|_{L(Y)}^{2}+1\right)}
$$

we can then write

with

$$
\frac{d}{d t} \rho_{\varepsilon}(t) \leq-a(\varepsilon)\|Y(t)\|_{X \times Y}^{2}
$$

$$
a(\varepsilon)=\varepsilon\left(1-\frac{\alpha}{2}\left(\left\|P_{A}\right\|_{L(X)}^{2}+1\right)\right) .
$$

From the definition of $\rho_{\varepsilon}$ :

$$
\|Y(t)\|_{X \times Y}^{2} \geq\left(1+\varepsilon \max \left(\left\|P_{A}\right\|_{L(X)},\left\|C^{-1}\right\|_{L(Y)}\right)\right)^{-1} \rho_{\varepsilon}(t)
$$

and the two last inequalities give

$$
\frac{d}{d t} \rho_{\varepsilon}(t) \leq-D(\varepsilon) \rho_{\varepsilon}(t),
$$

where

$$
D(\varepsilon)=\frac{a(\varepsilon)}{1+\varepsilon \max \left(\left\|P_{A}\right\|_{L(X)},\left\|C^{-1}\right\|_{L(Y)}\right)} .
$$

This proves the exponential decay of $\rho_{\varepsilon}(t)$.

Lemma 4. For $\varepsilon>0$, the application

$\varsigma_{\varepsilon}:\left\{\begin{array}{l}X \times Y \rightarrow R^{+} \\ Y=(u, v) \rightarrow \varsigma_{\varepsilon}(Y)=\left(\|Y\|_{X \times Y}^{2}+\varepsilon\left\langle P_{A} u, u\right\rangle_{X}+\varepsilon\left\langle-C^{-1} v, v\right\rangle_{Y}\right)^{\frac{1}{2}}\end{array}\right.$

defines a norm on $X \times Y$ which is equivalent to $\|Y\|_{X \times Y}$. 
Proof. One has the inequalities

$$
\|Y\|_{X \times Y} \leq \varsigma_{\varepsilon}(Y) \leq\left(1+\varepsilon \max \left(\left\|P_{A}\right\|_{L(X)},\left\|C^{-1}\right\|_{L(Y)}\right)\right)^{\frac{1}{2}}\|Y\|_{X \times Y} .
$$

The first inequality is obvious. The second is (4).

Proof of the theorem. Lemmas 3 and 4 prove the theorem since we deduce from them that, $\forall t \geq 0$, we have

$$
\|Y(t)\|_{X \times Y}^{2} \leq\left(1+\varepsilon \max \left(\left\|P_{A}\right\|_{L(X)},\left\|C^{-1}\right\|_{L(Y)}\right)\right)\|Y(0)\|_{X \times Y}^{2} e^{-D(\varepsilon) t},
$$

which proves the exponential decay and gives an estimation of the decay rate.

\section{The Asymptotic Stability}

Definition 5. $A C_{0}$ semigroup $(S(t))_{t}$ in a Banach space $E$ is said to be asymptotically stable if

$$
\lim _{t \rightarrow+\infty} S(t) x=0 \quad \forall x \in E .
$$

In this section, the assumption (E.S) is replaced by

\section{Assumption (A.S)}

1) $(A, D(A))$ has a compact resolvent and is a generator of contraction semigroup $\left(S_{A}(t)\right)$ on the Hilbert space $X$;

2) $(C, D(C))$ has a compact resolvent, is self-adjoint on the Hilbert space $Y$ and generator of an exponentially stable $C_{0}$ - semigroup $\left(S_{C}(t)\right)$;

3) i) $D(C) \subset D(B)$ and there exist two constants $\alpha_{C}$ and $\delta_{C}$ with

$$
0 \leq \alpha_{C}<1, \quad \delta_{C} \geq 0,
$$

such that

$$
\|B v\|_{X} \leq \alpha_{C}\|C v\|_{Y}+\delta_{C}\|v\|_{Y}
$$

ii)

$$
D(A) \subset D\left(B^{*}\right)
$$

and there exist two constants $\alpha_{A}$ and $\delta_{A}$ with

$$
0 \leq \alpha_{A}<1, \quad \delta_{A} \geq 0
$$

such that

$$
\left\|B^{*} u\right\|_{Y} \leq \alpha_{A}\|A u\|_{X}+\delta_{A}\|u\|_{X}
$$

Remark 6. The assumption $\left(A . S_{3}\right)$ is natural because the operator $L$ can be written as follows

$$
L=D+E
$$

with

$$
D=\left(\begin{array}{cc}
A & 0 \\
0 & C
\end{array}\right), \quad E=\left(\begin{array}{cc}
0 & B \\
-B^{*} & 0
\end{array}\right) .
$$

Clearly $D$ is a generator of contraction semigroup and since $E$ is dissipative, $L$ is also a generator of contraction semigroup (see for example [10], Corollary 3.3, p. 82). 
For

$$
Y_{0}=\left[\begin{array}{l}
u_{0} \\
v_{0}
\end{array}\right] \in X \times Y,
$$

let us denote by $\gamma\left(Y_{0}\right)$ its orbit and by $\omega\left(Y_{0}\right)$ its $\omega$ - limit set :

$$
\left\{\begin{array}{l}
\gamma\left(Y_{0}\right) \equiv\left\{S(t) Y_{0}, t \geq 0\right\}, \\
\omega\left(Y_{0}\right) \equiv\left\{Z \in X \times Y, \exists t_{n} \in R^{+}, t_{n} \rightarrow+\infty, Z=\lim _{t_{n} \rightarrow+\infty} S\left(t_{n}\right) Y_{0}\right\}
\end{array}\right.
$$

Proposition 7. If $Y_{0} \in D(L)$, then $\left(u^{\prime}, v^{\prime}\right)$ is bounded in $X \times Y$.

Proof. Letting $Y_{0} \in D\left(L^{2}\right)$, we obtain

$$
\left\{\begin{array}{l}
u^{\prime \prime}=A u^{\prime}+B v^{\prime} \\
v^{\prime \prime}=-B^{*} v^{\prime}+C v^{\prime}
\end{array}\right.
$$

with

$$
\left\{\begin{array}{l}
u^{\prime}(0)=A u_{0}+B v_{0} \\
v^{\prime}(0)=-B^{*} u_{0}+C v_{0}
\end{array}\right.
$$

So,

$$
\begin{aligned}
\left\|u^{\prime}\right\|_{X}^{2}+\left\|v^{\prime}\right\|_{Y}^{2} & \leq\left\|u^{\prime}(0)\right\|_{X}^{2}+\left\|v^{\prime}(0)\right\|_{Y}^{2} \\
& \leq\left\|A u_{0}+B v_{0}\right\|_{X}^{2}+\left\|-B^{*} u_{0}+C v_{0}\right\|_{Y}^{2} \\
& \leq 2\left[\left\|A u_{0}\right\|_{X}^{2}+\left\|B v_{0}\right\|_{X}^{2}+\left\|B^{*} u_{0}\right\|_{Y}^{2}+\left\|C v_{0}\right\|_{Y}^{2}\right]
\end{aligned}
$$

and, using the assumption $\left(A . S_{3}\right)$, we get

$$
\left\|u^{\prime}\right\|_{X}^{2}+\left\|v^{\prime}\right\|_{Y}^{2} \leq K\left[\left\|A u_{0}\right\|_{X}^{2}+\left\|v_{0}\right\|_{Y}^{2}+\left\|u_{0}\right\|_{X}^{2}+\left\|C v_{0}\right\|_{Y}^{2}\right],
$$

where $K$ is a positive constant. This proves the proposition for $Y_{0} \in D\left(L^{2}\right)$ and, by a density argument, we obtain the result for all $Y_{0} \in D(L)$.

Corollary 8. For all $Y_{0} \in D(L), \gamma\left(Y_{0}\right)$ is relatively compact in $X \times Y$.

Proof. One has

$$
\begin{gathered}
\|A u\|_{X} \leq\left\|u^{\prime}\right\|_{X}+\|B v\|_{X} \leq\left\|u^{\prime}\right\|_{X}+\delta_{C}\|v\|_{Y}+\alpha_{C}\|C v\|_{Y} \\
\|C v\|_{Y} \leq\left\|v^{\prime}\right\|_{Y}+\left\|B^{*} u\right\|_{Y} \leq\left\|v^{\prime}\right\|_{Y}+\delta_{A}\|u\|_{X}+\alpha_{A}\|A u\|_{X},
\end{gathered}
$$

So

$$
\left(1-\alpha_{A}\right)\|A u\|_{X}+\left(1-\alpha_{C}\right)\|C v\|_{Y} \leq\left\|u^{\prime}\right\|_{X}+\left\|v^{\prime}\right\|_{Y}+\delta_{A}\|u\|_{X}+\delta_{C}\|v\|_{Y} .
$$

Since we assumed that the constants $\alpha_{A}$ and $\alpha_{C}$ are less than one, from the last proposition we deduce that $\|A u\|_{X}$ and $\|C v\|_{Y}$ are bounded in $X$ and $Y$ respectively. As $A$ and $C$ have compact resolvent, we have the result.

We can then state 
Theorem 9. Under the assumption (A.S), if the unique solution of the system

$$
\left\{\begin{array}{l}
u^{\prime}=A u, \\
B^{*} u=0, \quad t \geq 0,
\end{array}\right.
$$

is $u \equiv 0$, then $S_{L}(t)$ is asymptotically stable.

Proof. Let $Y_{0} \in D\left(L^{2}\right)$ and $Y \in \omega\left(Y_{0}\right)$. Clearly,

$$
S_{L}(t) Y \in \omega\left(Y_{0}\right) \quad \forall t \geq 0,
$$

and

$$
\left\|S_{L}(t) Y\right\|_{X \times Y}^{2}=\|Y\|_{X \times Y}^{2} \quad \forall t \geq 0 .
$$

Now, let $\left(t_{n}\right)$ be such that

$$
S_{L}\left(t_{n}\right) Y_{0} \longrightarrow Y \quad \text { as } \quad n \rightarrow \infty .
$$

Since $S_{L}\left(t_{n}\right) L Y_{0}$ is relatively compact, there exists a subsequence $\left(t_{n_{j}}\right)$ such that

$$
L S_{L}\left(t_{n_{j}}\right) Y_{0}=S_{L}\left(t_{n_{j}}\right) L Y_{0} \longrightarrow Z \quad \text { as } \quad j \rightarrow \infty .
$$

Since $L$ is closed, $Y \in D(L)$ and $L Y=Z$. If $\left[\begin{array}{c}u(t) \\ v(t)\end{array}\right]=S_{L}(t) Y$, then we can write

$$
\frac{d}{d t}\left\|S_{L}(t) Y\right\|_{X \times Y}^{2}=R e\left((A u(t), u(t))_{X}+(C v(t), v(t))\right)_{Y}=0 .
$$

Thus, $v \equiv 0$ and $u$ verifies

$$
u^{\prime}=A u, \quad B^{*} u=0 .
$$

The assumption of the theorem implies that $u \equiv 0$. This proves that

$$
S_{L}(t) Y=0 \quad \forall t \geq 0 .
$$

Consequently, $Y=0$ and $\omega\left(Y_{0}\right)=\{0\}$. Since $S_{L}(t)$ is uniformly bounded and $D\left(L^{2}\right)$ is dense in $X \times Y$, the result follows.

\section{Applications}

As a first example, consider the problem

$$
\begin{cases}u^{\prime \prime}=\Delta u-a(x) u^{\prime}+b w, & \Omega \times R^{+}, \\ w^{\prime}=\Delta w-b^{*} u^{\prime}, & \Omega \times R^{+}, \\ u_{\partial \Omega}=w_{\partial \Omega}=0, & R^{+}, \\ u(0)=u_{0}, u^{\prime}(0)=u_{1}, w(0)=w_{0}, & \Omega,\end{cases}
$$

where $\Omega$ is an open bounded subset of $R^{n}$ with a smooth boundary, $a \in$ $L^{\infty}(\Omega)$ and $(b, D(b))$ is a linear operator on $L^{2}(\Omega)$ which will be precisely specified later. The system $(7)$ can be written formally as follows:

$$
\left\{\begin{array}{c}
Y^{\prime}=L Y \\
Y(0)=Y_{0}=\left[\begin{array}{c}
u_{0} \\
u_{1} \\
w_{0}
\end{array}\right]
\end{array}\right.
$$


with

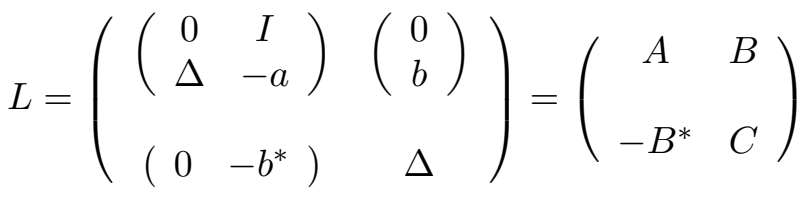

defined on $H=H_{0}^{1}(\Omega) \times L^{2}(\Omega) \times L^{2}(\Omega)$ (the energy space equipped with the product norm denoted by $\|\|$.$) and$

$$
D(L)=D(A) \times D(C)=\left(H^{2}(\Omega) \cap H_{0}^{1}(\Omega) \times H_{0}^{1}(\Omega)\right) \times\left(H^{2}(\Omega) \cap H_{0}^{1}(\Omega)\right) .
$$

Then, assuming the following geometrical control property (see [4])

$\Omega_{0} \subset \Omega$ is open and there exists $T>0$ with the property that every geodesic (ray) of length $T$ meets $\Omega_{0}$.

As a direct consequence of the results of the previous sections, one has

Theorem 10. The following statements are true:

(i) If a is nonnegative on $\Omega$ and

$$
a \geq a_{0}>0 \text { a.e on } \Omega_{0} \subset \Omega \text {, }
$$

where $\Omega_{0}$ is open and satisfies the geometrical control property and $b$ is $(-\Delta)^{\frac{1}{2}}$-bounded, then $\left(S_{L}(t)\right)_{t \geq 0}$ is exponentially stable: there exist $\omega>0$ and $M>0$ such that

$$
\left\|S_{L}(t)\right\| \leq M e^{-\omega t} \quad \text { for all } t \geq 0 ;
$$

(ii) If $b$ is $(-\Delta)^{\frac{1}{2}}$-bounded and $a \equiv 0$ a.e. in $\Omega$, then, for all $Y_{0} \in X$,

$$
\left\|S_{L}(t) Y_{0}\right\| \rightarrow 0 \quad \text { as } t \rightarrow+\infty .
$$

Proof. From the results of [4], $A$ generates an exponentially stable semigroup in case (i). The other assumptions of theorem 2 are easily derived. Property (ii) is a direct consequence of theorem 9 .

Remark 11. (a) This result is true, in particular, if $b$ is a bounded operator in $L^{2}(\Omega)$. In this case, one cannot expect exponential stability if $a \equiv 0$ and $b$ is a constant and, in a sense, (ii) in theorem 10 is optimal. More precisely, when $a$ and $b$ are constants, we have (see [1] and [2])

Proposition 12. The eigenvalues $\left(\lambda_{3 k+j}\right)$ of L satisfy the following properties

(i) for sufficiently large $k$, one of the eigenvalues (denoted by $\lambda_{3 k}$ ) is real and the two others are complex;

(ii) $\lambda_{3 k}>-\mu_{k}, \quad \operatorname{Re}\left(\lambda_{3 k+j}\right)<-\frac{a}{2}$ for all $k$ and $j=1,2$

$$
\lim _{k \rightarrow+\infty} \operatorname{Re}\left(\lambda_{3 k}\right)=-\infty, \quad \lim _{k \rightarrow+\infty} \operatorname{Re}\left(\lambda_{3 k+j}\right)=-\frac{a}{2} \quad j=1,2
$$


(iii) L satisfies the spectrum determined growth assumption and for every $b \in R$, there exists $\left.a_{0}=a_{0}(b) \in\right] 0, \sqrt{2 \mu_{1}}[$ such that

$$
\begin{aligned}
\bar{\omega}(L) \quad & :=\inf \left\{\omega \in R, \exists M>0,\left\|S_{L}(t)\right\| \leq M e^{\omega t}\right\} \\
& \left.=\bar{\omega}(\tilde{A}) \quad \text { if } a \in] 0, a_{0}\right], \\
\bar{\omega}(L) & >\bar{\omega}(\tilde{A}) \quad \text { if } a>a_{0},
\end{aligned}
$$

where the $\mu_{k}$ are the eigenvalues of $(-\Delta)$ and

$$
\tilde{A}=\left(\begin{array}{cc}
0 & I \\
\Delta & -a
\end{array}\right) ; \quad \bar{\omega}(\tilde{A})=\left\{\begin{array}{l}
-\frac{a}{2} \quad \text { if } a \leq \sqrt{2 \mu_{1}}, \\
-\frac{a}{2}+\frac{\sqrt{a^{2}-4 \mu_{1}}}{2} \quad \text { if } a>\sqrt{2 \mu_{1}} .
\end{array}\right.
$$

(b) If one takes $b=d \cdot \nabla$, where $d$ is a vector of $R^{n}$, the result still holds. The interest here is that, in the one-dimensional case (the one-dimensional thermoelasticity system), the exponential stability remains even if a $\equiv 0$ (see [8]).

The second example deals with the boundary stabilization of the thermoelasticity system. Let $\Omega$ an open bounded set in $R^{n}$ with smooth boundary $\Gamma$,

$$
\begin{cases}u^{\prime \prime}=\Delta u+\alpha \nabla w, & R^{+} \times \Omega, \\ w^{\prime}=\Delta w+\alpha \nabla \cdot u^{\prime}, & R^{+} \times \Omega, \\ \frac{\partial u}{\partial \nu}=b u^{\prime}, & R^{+} \times \Gamma_{0}, \\ u=0, & R^{+} \times \Gamma_{1}, \\ w=0, & R^{+} \times \Gamma, \\ u(0)=u_{0}, u^{\prime}(0)=u_{1}, w(0)=w_{0}, & \Omega .\end{cases}
$$

where $u=\left[\left(u_{1}, \ldots, u_{n}\right)\right]$ (resp. $\left.w\right)$ is the displacement (resp. the temperature) of the system, $\nu(x)$ is the exterior normal to $\Gamma$ at $x, \alpha>0$ is the coupling parameter and $x_{0}$ is a fixed point in $R^{n}$ ( $n \leq 3$ for physical cases) (see [7]),

$$
\begin{gathered}
m(x)=x-x_{0} \quad x \in R^{n}, \\
\Gamma_{0}=\{x \in \Gamma ; m(x) \cdot \nu(x)>0\}, \\
\Gamma_{1}=\Gamma-\Gamma_{0}, \\
b(x)=-m(x) \cdot \nu(x) .
\end{gathered}
$$

We assume that

$$
\Gamma_{1} \neq \emptyset
$$


and set

$$
H_{\Gamma_{1}}^{1}(\Omega)=\left\{u \in H^{1}(\Omega) ; u=0 \text { on } \Gamma_{1}\right\} .
$$

On this space, we define the scalar product

$$
<u, v>=\int_{\Omega} \nabla u . \nabla v d x .
$$

The energy space will be the Hilbert space

$$
H=\left(H_{\Gamma_{1}}^{1}(\Omega)\right)^{n} \times\left(L^{2}(\Omega)\right)^{n} \times L^{2}(\Omega)
$$

with the scalar product

$$
<\left[\begin{array}{c}
u \\
v \\
w
\end{array}\right],\left[\begin{array}{l}
f \\
g \\
h
\end{array}\right]>=\int_{\Omega}(\nabla u \cdot \nabla f+v g+w h) d x
$$

and we denote by $\|\cdot\|$ the induced norm on $H$. |.| will denote the $L^{2}$-norm.

Let

$$
\begin{aligned}
& L=\left(\begin{array}{cc}
\left.\left(\begin{array}{cc}
0 & I \\
\Delta & 0
\end{array}\right)\left(\begin{array}{c}
0 \\
\alpha \nabla
\end{array}\right)\right)=\left(\begin{array}{cc}
A & B \\
-B^{*} & C
\end{array}\right), \\
\left(\begin{array}{cc}
0 & \alpha \nabla \cdot)
\end{array}\right)
\end{array}\right) \\
& D(L)= D(A) \times D(C) \\
&=\left\{\left[\begin{array}{l}
u \\
v
\end{array}\right] \in H_{\Gamma_{1}}^{1}(\Omega)^{n} \times L^{2}(\Omega)^{n} ; \Delta u \in\left(L^{2}(\Omega)\right)^{n}, v \in H_{\Gamma_{1}}^{1}(\Omega)^{n}, \frac{\partial u}{\partial \nu}\right. \\
&\left.=\text { bv on } \Gamma_{0}\right\} \times\left(H^{2}(\Omega) \cap H_{0}^{1}(\Omega)\right) .
\end{aligned}
$$

One has the following: Assumption (E.S) is satisfied if a geometrical hypothesis on $\Omega$ (see below in the proof) is added which insures the validity of Grisvard's integral formula.

Proof. Following Grisvard [5], in the case $n=2$ one has the Green formula:

$$
\begin{aligned}
\int_{\Omega} \Delta u m \cdot \nabla u d x & =\frac{1}{2} \int_{\Gamma_{1}} m \cdot \nu\left|\frac{\partial u}{\partial \nu}\right|^{2} d \sigma-\frac{1}{2} \int_{\Gamma_{0}} m \cdot \nu\left|\frac{\partial u}{\partial \tau}\right|^{2} d \sigma \\
& +\left(\frac{\Pi}{8}\right) \sum_{S \in \Sigma^{S}} c_{S}^{2} m(S) \cdot \tau_{S}-\left(\frac{\Pi}{8}\right) \sum_{S \in \Sigma^{C}} c_{S}^{2} m(S) \cdot \tau_{S}
\end{aligned}
$$

for all $u$ in $D(\Delta)$ (with mixed boundary conditions) where $u-\sum_{S \in \Sigma} c_{S} u_{S}$ is in $H^{2}(\Omega), u_{S}$ being the first singularities of the Laplace operator. In this formula, $\Sigma$ is a finite set (the "vertexes") and $\left(\Sigma^{c}, \Sigma^{s}\right)$ is a partition of $\Sigma$ defined by

(i) $S \in \Sigma^{c}$ if $S$ is downstream of $\Gamma_{1}$ following the positive orientation of $\Gamma$;

(ii) $S \in \Sigma^{s}$ if $S$ is downstream of $\Gamma_{0}$ following the positive orientation of $\Gamma$. In this case, the geometric condition on $\Omega$ is

$$
m \cdot \tau \geq 0 \text { on } \Sigma^{c} ; \quad m \cdot \tau \leq 0 \text { on } \Sigma^{s} .
$$


This last condition implies the inequality

$$
\int_{\Omega} \Delta u m \cdot \nabla u d x \leq \frac{1}{2} \int_{\Gamma_{1}} m \cdot \nu\left|\frac{\partial u}{\partial \nu}\right|^{2} d \sigma-\frac{1}{2} \int_{\Gamma_{0}} m \cdot \nu\left|\frac{\partial u}{\partial \tau}\right|^{2} d \sigma .
$$

The exponential stability of the semigroup whose generator is $A$ is then proved as in [6]. The operator $B$ is $(-\Delta)^{1 / 2}$-bounded and $D(A) \subset D\left(B^{*}\right)$. Note that the last (crucial) inequality remains true in higher dimension with similar geometric restriction on $\Omega$ (see [5] for $n=3$ and [M] for $n \geq 3$ ) and thus the semigroup is also exponentially stable.

Theorem 13. Under the previous assumption, $\left(S_{L}(t)\right)$ is exponentially stable.

We give, in what follows, a second proof, more constructive, which insures an estimate of $\omega_{L}$, the constant of the exponential decay of $S_{L}(t)$.

Proof. Let $Y=\left[\begin{array}{c}u \\ v \\ w\end{array}\right]$ a solution of (10). We define, for all $t \geq 0$, the function

$$
\rho_{\varepsilon}(t)=\frac{1}{2}\|Y(t)\|^{2}+\varepsilon\left(\sum_{i=1}^{n} \rho_{1}^{i}(t)+\rho_{2}(t)\right),
$$

where

$$
\rho_{1}^{i}(t)=\int_{\Omega} v_{i}(t, x)\left[2 m \cdot \nabla u_{i}(t, x)+(n-1) u_{i}(t, x)\right] d x, \quad i=1, \ldots, n,
$$

and

$$
\rho_{2}(t)=\frac{1}{2}\left|\nabla \Delta^{-1} w(t, x)\right|^{2} .
$$

$\Delta^{-1}$ is the inverse of $\Delta$ considered as an operator of $L^{2}(\Omega)$ with domain $D(\Delta)=H^{2}(\Omega) \cap H_{0}^{1}(\Omega)$. Differentiating $\rho_{1}^{i}$, we obtain

$$
\begin{aligned}
& \frac{d}{d t} \rho_{1}^{i}=\int_{\Omega} v_{i}^{\prime}\left[2 m \cdot \nabla u_{i}+(n-1) u_{i}\right]+v_{i}\left[2 m \cdot \nabla v_{i}+(n-1) v_{i}\right] d x \\
& =\int_{\Omega}\left(\Delta u_{i}+\alpha \frac{\partial w}{\partial x_{i}}\right)\left[2 m \cdot \nabla u_{i}+(n-1) u_{i}\right]+v_{i}\left[2 m \cdot \nabla v_{i}+(n-1) v_{i}\right] d x
\end{aligned}
$$

As in $([6],(2.20)-(2.22))$, we have, for all $i=1, \ldots, n$,

$$
\begin{aligned}
(n-1) \int_{\Omega} u_{i} \Delta u_{i} d x & =-(n-1) \int_{\Gamma_{0}}(m \cdot \nu) v_{i} u_{i} d \Gamma-(n-1) \int_{\Omega}\left|\nabla u_{i}\right|^{2} d x \\
2 \int_{\Omega} v_{i} m \cdot \nabla v_{i} d x & =\int_{\Gamma_{0}} m \cdot \nu\left|v_{i}\right|^{2} d \Gamma-n \int_{\Omega}\left|v_{i}\right|^{2} d x \\
2 \int_{\Omega}\left(\Delta u_{i}\right)\left(m \cdot \nabla u_{i}\right) d x \leq & (n-2) \int_{\Omega}\left|\nabla u_{i}\right|^{2} d x \\
& -\int_{\Gamma_{0}}(m \cdot \nu)\left[2 v_{i}\left(m \cdot \nabla u_{i}\right)+\left|\nabla u_{i}\right|^{2}\right] d \Gamma .
\end{aligned}
$$


From these three last relations, we get

$$
\begin{aligned}
\frac{d}{d t} \rho_{1}^{i} \leq & -\left|\nabla u_{i}\right|^{2}-\left|v_{i}\right|^{2}+\int_{\Omega} \alpha \frac{\partial w}{\partial x_{i}}\left[2 m \cdot \nabla u_{i}+(n-1) u_{i}\right] d x \\
& -\int_{\Gamma_{0}} m \cdot \nu\left[2 v_{i} m \cdot \nabla u_{i}+\left|\nabla u_{i}\right|^{2}-\left|v_{i}\right|^{2}+(n-1) u_{i} v_{i}\right] d \Gamma .
\end{aligned}
$$

On the other hand,

$$
\begin{aligned}
\frac{d}{d t} \rho_{2} & =\int_{\Omega}\left(\nabla \Delta^{-1} w\right) \cdot\left(\nabla \Delta^{-1} w^{\prime}\right) d x \\
& =-\int_{\Omega}\left(\Delta^{-1} w\right) w^{\prime} d x
\end{aligned}
$$

and then, using the second equation in (10), we obtain

$$
\frac{d}{d t} \rho_{2}=-|w|^{2}-\int_{\Omega} \alpha(\nabla \cdot v)\left(\Delta^{-1} w\right) d x .
$$

Noting that

one obtains

$$
\frac{1}{2} \frac{d}{d t}\|Y(t)\|^{2}=(L Y, Y)
$$

$$
\frac{1}{2} \frac{d}{d t}\|Y(t)\|^{2}=-\int_{\Gamma_{0}} m \cdot \nu|v|^{2} d \Gamma-\int_{\Omega}|\nabla w|^{2} d x .
$$

Thus, from (12)-(14),

$$
\begin{aligned}
\frac{d}{d t} \rho_{\varepsilon} \leq & -\int_{\Gamma_{0}} m \cdot \nu\left[(1-\varepsilon)|v|^{2}+\varepsilon(n-1) u \cdot v\right. \\
& \left.+2 \varepsilon v \cdot(m \mid \nabla) u+\varepsilon|\nabla u|^{2}\right] d \Gamma \\
& +\varepsilon \int_{\Omega} \alpha \nabla w \cdot[2(m \mid \nabla) u+(n-1) u] d x+ \\
& +\varepsilon \int_{\Omega} \alpha(\nabla \cdot v)\left(\Delta^{-1} w\right) d x-\varepsilon\|Y\|^{2}-|\nabla w|^{2}
\end{aligned}
$$

where, in this last inequality, we used the notation

$$
(m \mid \nabla) u=\left[\begin{array}{c}
m \cdot \nabla u_{1} \\
\cdot \\
\cdot \\
\cdot \dot{v} u_{n}
\end{array}\right] .
$$

Let $R=\|m\|_{L^{\infty}(\Omega)}, C_{0}$ the Poincaré constant in $\widetilde{H_{0}^{1}}(\Omega)$ and $C_{1}$ defined by

$$
\int_{\Gamma_{0}} m \cdot \nu \quad|u|^{2} d \Gamma \leq C_{1}|\nabla u|^{2} \quad \forall u \in \widetilde{H_{0}^{1}}(\Omega) .
$$

Let us denote by $I_{0}$ the integral on $\Gamma_{0}$ in inequality (15). It is estimated as in [6] by

$$
\begin{aligned}
-I_{0} \leq & -\left[1-\varepsilon\left(1+R^{2}+\frac{(n-1)^{2} C_{1}}{2}\right)\right] \int_{\Gamma_{0}} m \cdot \nu|v|^{2} d \Gamma \\
& +\frac{\varepsilon}{2}\left(|\nabla u|^{2}+|v|^{2}\right) .
\end{aligned}
$$


For the next terms of (15), let $\beta>0$ any constant. One has

$$
\left|\int_{\Omega} \alpha \nabla w \cdot(m \mid \nabla) u d x\right| \leq \frac{|\alpha|^{2}}{2 \beta}|\nabla w|^{2}+\frac{\beta}{2} R^{2}|\nabla u|^{2}
$$

and

$$
\left|\int_{\Omega} \alpha \nabla w \cdot(n-1) u d x\right| \leq \frac{|\alpha|^{2}}{2 \beta}|\nabla w|^{2}+\frac{\beta(n-1)^{2} C_{0}}{2}|\nabla u|^{2} .
$$

Using first Green's formula, we obtain for the third integral

$$
\left|\int_{\Omega} \alpha(\nabla \cdot v)\left(\Delta^{-1} w\right) d x\right| \leq \frac{|\alpha|^{2} C_{0}^{2}}{2 \beta}|\nabla w|^{2}+\frac{\beta}{2}|v|^{2} .
$$

With the inequalities (16)-(19), we conclude that

$$
\begin{aligned}
\frac{d}{d t} \rho_{\varepsilon} \leq & -\varepsilon\|Y\|^{2}+\left[\frac{|\alpha|^{2}}{2 \beta} \varepsilon\left(2+C_{0}^{2}\right)-1\right]|\nabla w|^{2} \\
& -\left[1-\varepsilon\left(1+R^{2}+\frac{(n-1)^{2} C_{1}}{2}\right)\right] \int_{\Gamma_{0}} m \cdot \nu|v|^{2} d \Gamma \\
& +\frac{\varepsilon}{2}\left(1+\beta\left[R^{2}+(n-1)^{2} C_{0}\right]\right)|\nabla u|^{2}+\varepsilon \frac{\beta+1}{2}|v|^{2} .
\end{aligned}
$$

We choose $\beta>0$ such that

$$
1-\beta\left[R^{2}+(n-1)^{2} C_{0}\right]>0 ; \quad 1-\beta>0
$$

and $\varepsilon>0$ in such a way that

$$
\varepsilon \frac{|\alpha|^{2}}{2 \beta}\left(2+C_{0}^{2}\right)-1 \leq 0 ; \quad 1-\varepsilon\left(1+R^{2}+\frac{(n-1)^{2} C_{1}}{2}\right) \geq 0 .
$$

Since $m \cdot \nu \geq 0$ on $\Gamma_{0}$, one derives the inequality

$$
\frac{d}{d t} \rho_{\varepsilon} \leq-\varepsilon C_{2}\|Y\|^{2}
$$

with

$$
C_{2}\left(=C_{2}(\beta)\right)=\min \left(1-\beta\left[R^{2}+(n-1)^{2} C_{0}\right], 1-\beta\right) .
$$

But, on the other hand, for all $t \geq 0$,

$$
\rho_{\varepsilon}(t) \leq\left(\frac{1}{2}+\frac{1}{2} \varepsilon \max \left(C_{0}, 2 R+(n-1) C_{0}\right)\right)\|Y(t)\|^{2} .
$$

As

$$
\rho_{\varepsilon}(t) \geq\left(\frac{1}{2}-\frac{\varepsilon}{2}\left(2 R+(n-1) C_{0}\right)\right)\|Y(t)\|^{2},
$$

we get the result with

$$
2 \omega \geq \frac{\varepsilon C_{2}(\beta)}{\left(\frac{1}{2}+\frac{1}{2} \varepsilon \max \left(C_{0}, 2 R+(n-1) C_{0}\right)\right)}
$$

and $\varepsilon$ verifying the condition: $\left(1-\varepsilon\left(2 R+(n-1) C_{0}\right)>0\right.$. 


\section{REFERENCES}

[1] F. Ammar Khodja and A. Benabdallah, Stabilisation de l'équation des ondes par un contrôleur dynamique, (French), C. R. Acad. Sci. Paris Sér. I Math. 321 (1995), 195-198.

[2] F. Ammar Khodja and A. Benabdallah, Stabilization of the wave equation by a dynamical controller, (French), Prépub. de l'Équipe de Mathématiques de Besançon, \#22, 1995.

[3] F. Ammar Khodja, A. Benabdallah and D. Teniou, Stabilisation frontière et interne d'un système similaire à celui de la thermoélasticité, (French), C. R. Acad. Sci Paris Sér. I Math. 322 (1996), 551-556.

[4] C. Bardos, G. Lebeau and J. Rauch, Sharp sufficient conditions for the observation, control and stabilization of waves from the boundary, SIAM J. Control Optim. 30 (1992), 1024-1065.

[5] P. Grisvard, Contrôlabilité exacte des solutions de l'équation des ondes en présence de singularités, J. Math. Pures Appl. (9) 68 (1989), 215-219.

[6] V. Komornik and E. Zuazua, A direct method for the boundary stabilization of the wave equation, J. Math. Pures Appl. (9) 69 (1990), 33-54.

[7] J.-L. Lions, Contrôlabilité Exacte, Perturbations et Stabilisation de Systèmes Distribués, (French), \#1 and \#2, Recherches en Mathématiques Appliquées, \#8 and \#9, Masson, Paris, 1988.

[8] Z. Liu and S. Zheng, Exponential stability of the semigroup associated with a thermoelastic system, Quart. Appl. Math. 2 (1993), 535-545.

[9] M. Moussaoui, Singularités des solutions du problème mêlé, contrôlabilité exacte et stabilisation frontière, Esaim Proc. 1 (1996), 157-168.

[10] A. Pazy, Semigroups of Linear Operators and Applications to Partial Differential Equations, Applied Mathematical Sciences, \#44, Springer-Verlag, New York-Berlin, 1983.

[11] A. Pritchard and J. Zabczyk, Stability and stabilizability of infinite dimensional systems, SIAM Rev. 23 (1981), 25-61.

Farid Ammar Khodja and Assia Benabdallah

Laboratoire de Calcul Scientifique

Groupe Matériaux Intelligents

URA C.N.R.S. 741

16, Route De Gray

25030 Besançon Cedex, FRANCE

E-mail address: abenabda@utinam.univ-fcomte.fr

Djamel Teniou

Institut DE MAThÉmatiques

U.S.T.H.B.- B.P. 32 - 16110 ALGERIA 


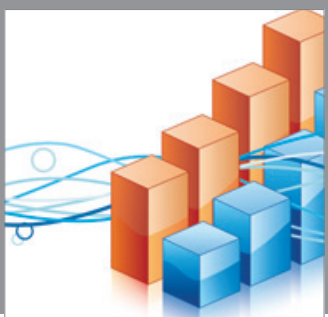

Advances in

Operations Research

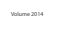

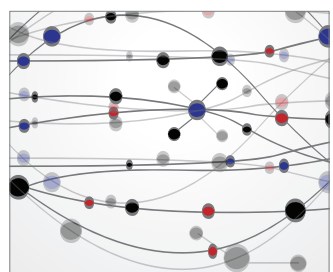

\section{The Scientific} World Journal
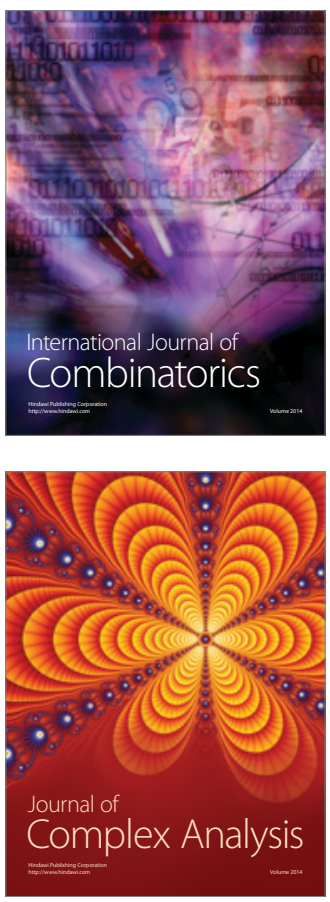

International Journal of

Mathematics and

Mathematical

Sciences
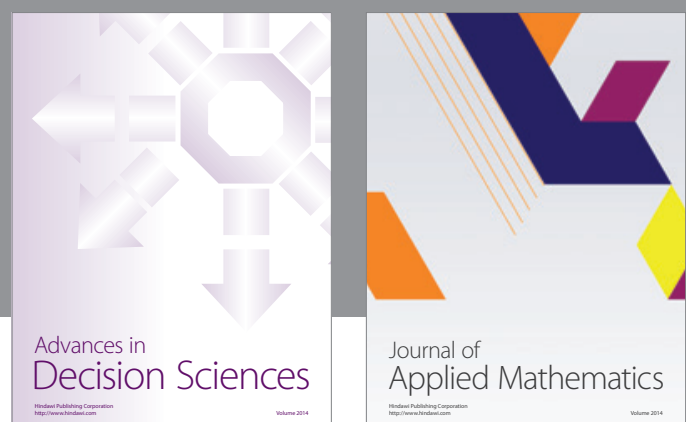

Journal of

Applied Mathematics
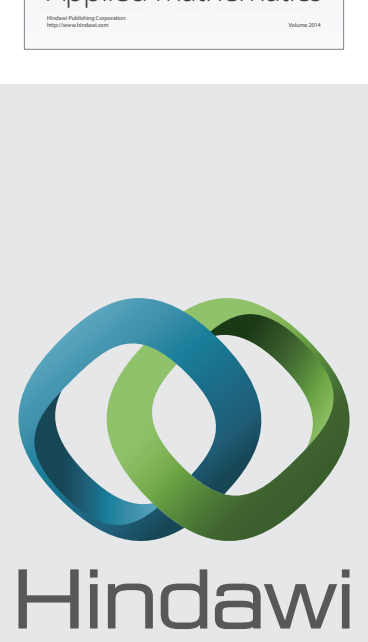

Submit your manuscripts at http://www.hindawi.com
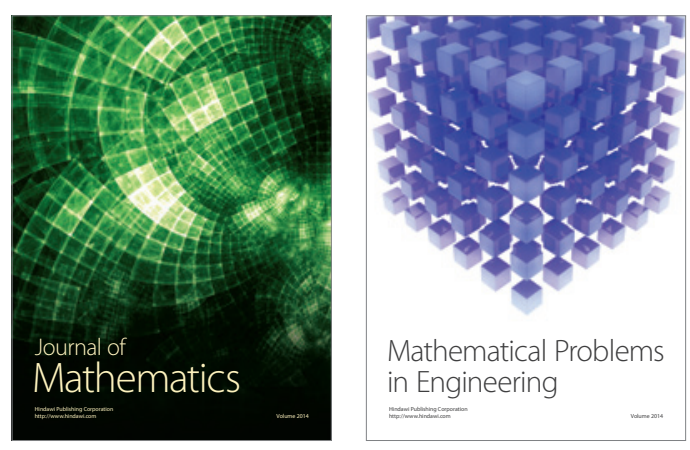

Mathematical Problems in Engineering
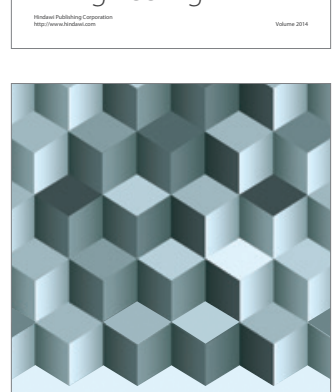

Journal of

Function Spaces
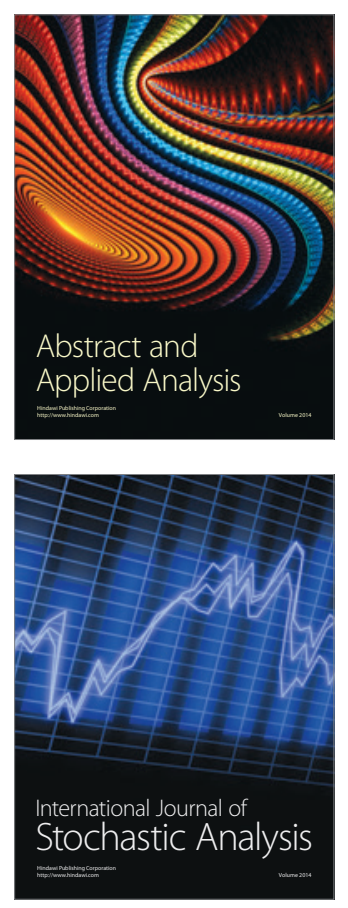

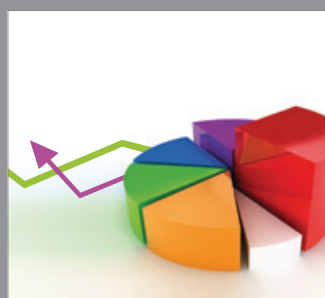

ournal of

Probability and Statistics

Promensencen
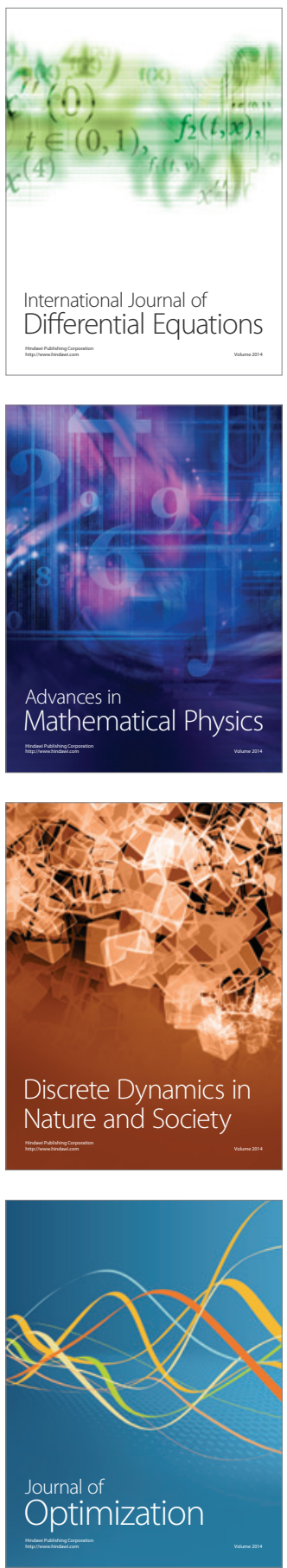